University of Nebraska - Lincoln

DigitalCommons@University of Nebraska - Lincoln

Biological Systems Engineering: Papers and

Publications

Biological Systems Engineering

2011

Effect of flow gradient, ROI size and random scatterer movement during speckle size estimation based blood flow measurement

Tiantian Xu

University of Nebraska-Lincoln, eason1984@gmail.com

Greg Bashford

University of Nebraska at Lincoln, gbashford2@unl.edu

Follow this and additional works at: https://digitalcommons.unl.edu/biosysengfacpub

Part of the Biological Engineering Commons

$\mathrm{Xu}$, Tiantian and Bashford, Greg, "Effect of flow gradient, ROI size and random scatterer movement during speckle size estimation based blood flow measurement" (2011). Biological Systems Engineering: Papers and Publications. 234.

https://digitalcommons.unl.edu/biosysengfacpub/234

This Article is brought to you for free and open access by the Biological Systems Engineering at DigitalCommons@University of Nebraska - Lincoln. It has been accepted for inclusion in Biological Systems Engineering: Papers and Publications by an authorized administrator of DigitalCommons@University of Nebraska Lincoln. 


\section{Effect of flow gradient, ROI size and random scatterer movement during speckle size estimation based blood flow measurement}

\author{
Tiantian Xu \\ Department of Biological Systems Engineering \\ University of Nebraska-Lincoln \\ Lincoln, NE, USA \\ eason1984@gmail.com
}

\author{
Gregory R. Bashford \\ Department of Biological Systems Engineering \\ University of Nebraska-Lincoln \\ Lincoln, NE, USA \\ gbashford2@unl.edu
}

\begin{abstract}
Conventional blood flow velocity measurement using ultrasound is capable of resolving the axial component (i.e., that aligned with the ultrasound propagation direction) of the blood flow velocity vector. However, these Doppler-based methods are incapable of detecting blood flow in the direction normal to the ultrasound beam. An algorithm which measures the lateral blood flow velocity using speckle size change with scan velocity was developed in our previous studies. This method uses the apparent speckle size change that occurs when scatterers are moving relative to the spatial rate of A-line acquisition. Our previous results showed that the estimation error of this algorithm increases with increasing flow gradient and random scatterer movement. In this paper, the relationship between the estimation performance and flow gradient, random scatterer movement and ROI size is investigated and quantitatively assessed. Simulated blood flow data with and without flow gradient and random scatterer movement were generated by the Field II simulation program. The flow gradient is introduced by a parabolic flow profile in the simulated vessel and the random scatterer movement is generated by adding Gaussian noise to the scatterers' position with a standard deviation as much as onetenth of the speckle cell size in each direction. Our results showed that: 1) in plug flow, estimation error decreases with increasing ROI size, with an average minimum error below $5 \%$. An optimal ROI size exists in both directions, which is 2.5 axial speckle cell lengths axially and 30 lateral speckle cell widths laterally; 2) the estimation error increases up to $10 \%$ with flow gradient; 3 ) an optimal lateral ROI size still exists given the presence of a flow gradient; 4) the estimation error increases with increasing axial ROI size since the correlation length shortens by the introduction of a flow gradient; 5) in addition to the previous results, when random scatterer movement is introduced into the blood flow, the average estimation error is worse by about a factor of three than data without random scatterer movement.
\end{abstract}

Keywords-blood flow detection; speckle size; scan velocity; flow gradient;

\section{INTRODUCTION}

Ultrasound has been widely used as a diagnostic tool in the cardiovascular system. It is known that the distribution of the blood velocities within a vessel contains valuable diagnostic information. Likewise, motion of the heart tissue is dependent on the health of cardiac muscles [1].
Currently, most quantitative flow measurement done in commercial ultrasound occurs along the scan axis, i.e., in the direction normal to the transducer face. This is because Doppler-based instruments cannot resolve flow parallel to the transducer face. If a method were devised that measured flow parallel to the transducer surface, then the two could be combined to resolve the two-dimensional velocity vector in the scan plane, providing better clinical information to a physician.

Previously, we showed that there is a linear relationship between apparent speckle size (due to A-line acquisition rate) and blood flow velocity [2]. Furthermore, our latest investigation of the blood flow measurement based on speckle size estimation showed that, qualitatively, the estimation error increases slightly with the flow gradient and random scatterer movement [3]. In this paper, specific quantitative relationships between flow gradient, random scatterer movement and estimation performance based on speckle size estimation were investigated. Also, the effect of ROI size on estimation performance was studied in this paper.

\section{BACKGROUND}

The Doppler effect in ultrasound (actually a measurement of phase change) is widely used in ultrasound to measure blood flow. Kasai et al. developed an algorithm to quickly estimate the mean velocity over a large spatial field of view based on an autocorrelation technique [4], which is now commonly referred to as color flow. A complementary method, referred to as spectral Doppler, is capable of estimating a velocity distribution at a small (resolution-limited) region of interest by displaying a spectral plot of the (temporal- and wall-filtered) flow signal [5].

However, Doppler is not able to measure the velocity vector projection along the lateral dimension of the ultrasound beam, since there is no Doppler frequency shift when the transducer is aligned parallel to the blood flow. Some researchers have formed alternative estimation algorithms to solve this problem. For example, estimating the transit time across the ultrasound beam was proposed for measuring flow parallel to the transducer face. One method described by Newhouse and Reid measures the variance of the Doppler signals returned from lateral flow [6]. The spatial quadrature technique was proposed to estimate lateral motion by employing a modulation in the acoustical field in the lateral 
direction $[7 ; 8]$. Direction and magnitude of local blood speckle pattern displacement using consecutive B-mode images were measured by Trahey et al, to predict lateral flow [9]. Feature tracking identified features of the ultrasonic speckle and followed them over time to detect motion in a pulse echo system $[10 ; 11]$. Sample tracking measured the time shift of each sample in a delayed echo signal with respect to a continuous, interpolated representation of the reference echo signal to provide time-delay estimation [12]. Maximum likelihood blood velocity estimation incorporated properties of flow physics into cross correlation to perform 2-D flow imaging [13]. A more complete review can be found in references [14] and [15]. These methods either use information from multiple ultrasound beam scan positions or require multiple images to form a flow estimate.

Based on a patent co-authored by one of the present authors (GRB) in 2001 [16], an algorithm which measures the lateral blood flow velocity using speckle size changes with scan velocity was developed in our previous studies $[2 ; 3 ; 17]$. This method uses the apparent speckle size change that occurs when scatterers are moving relative to the spatial rate of A-line acquisition. The speckle size was defined as the full-widthhalf-maximum (FWHM) of the auto-covariance (ACVF) of a region-of -interest (ROI) in the US B-mode (detected) data. We define the "scan velocity" to be the spatial rate at which individual ultrasound A-lines are collected laterally across the transducer [3].

\section{Materials AND Methods}

A Field II simulation [18; 19] was used to generate blood flow data for the experiments. The parameters of the transducer were set to match the V13-5 transducer (192 elements, 6.15 $\mathrm{MHz}$ center frequency) of the SONOLINE Antares ultrasound imaging system (Siemens Medical Solutions, Ultrasound Division, Issaquah, WA) in our laboratory. A blood flow phantom was also generated by computer simulation. The simulated phantom consisted of a lateral vessel $5 \mathrm{~mm}$ in diameter and positioned $20 \mathrm{~mm}$ from the surface of the transducer, which is the approximate size and location of a carotid artery branch. At least ten scatterers with random complex Gaussian reflection coefficients were randomly positioned (2D uniform distribution) in each speckle cell to produce "fully developed" speckle patterns [20]. The distance between each A-line is $0.1234 \mathrm{~mm}$ and 312 lines were simulated for each B-mode image. The scanning speed was set as $50 \mathrm{~cm} / \mathrm{s}$, corresponding to a B-mode PRF of $4052 \mathrm{~Hz}$, which is a typical value used in SONOLINE Antares ultrasound imaging.

According to typical blood flow velocities in the common carotid artery [21], four flow conditions were simulated: plug flow, parabolic flow, plug flow with random scatterer movement and parabolic flow with random scatterer movement.

To investigate the relationship between the estimation performance and ROI size, for the plug flow, the ROI was selected at the center of the vessel, the lateral ROI size was varied from 5 to 60 times the lateral speckle cell length and the axial ROI size was varied from 1 to 3 times the axial speckle cell width. In the parabolic flow, the ROI was positioned at three different sites where the distance from the vessel longitudinal axis was $1.35,0.8$ and $0 \mathrm{~mm}$. For each position, the lateral ROI size was varied from 5 to 60 times the lateral speckle cell length and the axial ROI size was varied from 1 to 3 times the axial speckle cell width.

To investigate the relationship between the estimation performance and the flow gradient, the ROI was positioned at seven sites within parabolic blood flow, where distance from the vessel longitudinal axis was $\pm 1.8, \pm 1.2, \pm 0.6$ and $0 \mathrm{~mm}$. The ROI size at these positions was fixed according to the information gained from the relationship between estimation performance and ROI size.

In addition to flow gradient in the blood flow, random movement of scatterers (red cells) occurs occasionally, especially in vessel bifurcation areas where turbulent flow potentially develops. Thus, all the analyses above were performed both on flow data with and without random scatterer movement. The effect of random scatterer movement on the estimation performance can be determined by comparing the estimation results of these two conditions.

\section{RESULTS}

The relationship between estimation performance and ROI size is shown in Fig. 1. Part (a) shows the relationship between the estimation error and axial ROI size and part (b) shows the relationship between the estimation error and lateral ROI size. Ten data sets were used for the estimation and the error bars show \pm one standard deviation of the estimation error. The first

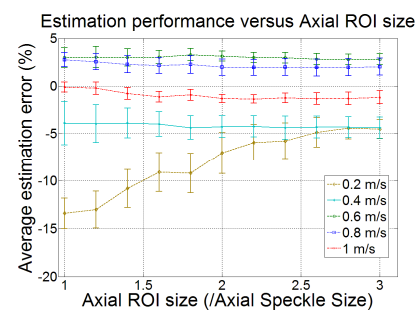

(a)

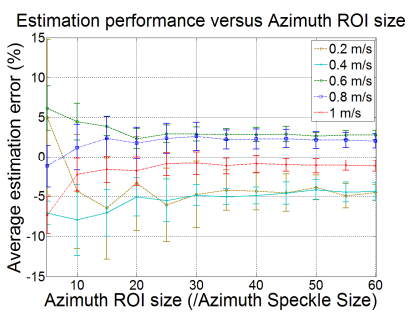

(b)
Fig.1. The relationship between estimation error and (a) axial ROI size, (b) lateral ROI size.

type of flow (plug flow, no random scatterer movement) is used for this result.

Fig. 2 shows the relationship between the estimation error and flow gradient. When the maximum velocity in the parabolic flow profile is $1 \mathrm{~m} / \mathrm{s}$, the maximum error is about $13 \%$ and the standard deviation is about $3 \%$. The minimum estimation error is about $1 \%$ and the standard deviation decreases to about $1 \%$. A similar relationship exists when the maximum velocity is $0.9 \mathrm{~m} / \mathrm{s}$. The estimation error decreases from $-5 \%$ to $1 \%$, and the standard deviation decreases from $3 \%$ to about $1 \%$ from vessel's boundary to the center. When the maximum velocity is $0.8 \mathrm{~m} / \mathrm{s}$, the estimation error decreases from $6 \%$ to $1 \%$, and the standard deviation decreases from $4 \%$ to about $1 \%$ from vessel's boundary to the center. 
The relationship between estimation error and ROI size was also investigated given the presence of a flow gradient, which can be seen in Fig. 3.

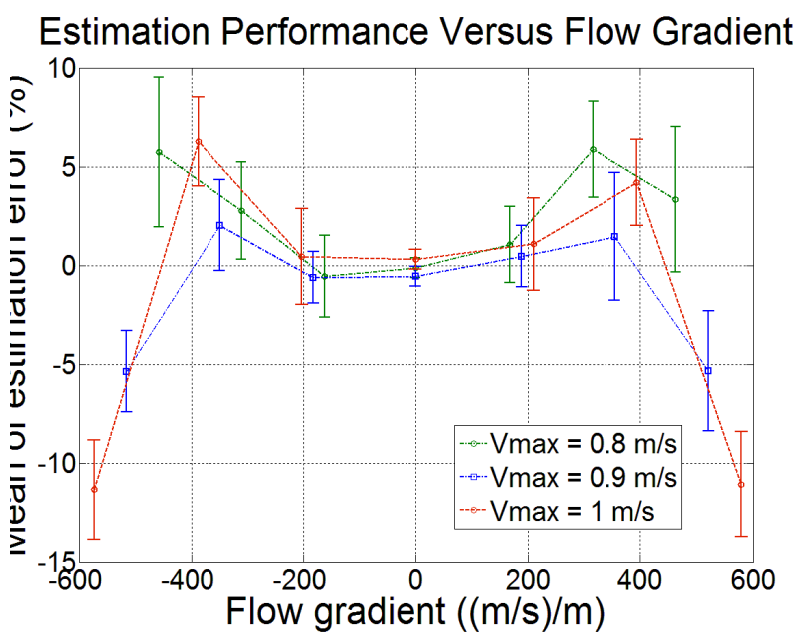

ig.2. The relationship between estimation error and flow gradient.



(a)

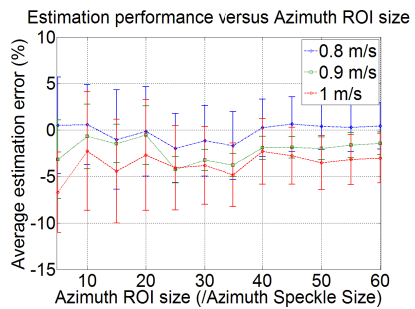

(c)

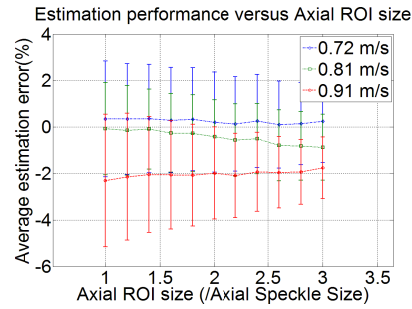

(e)

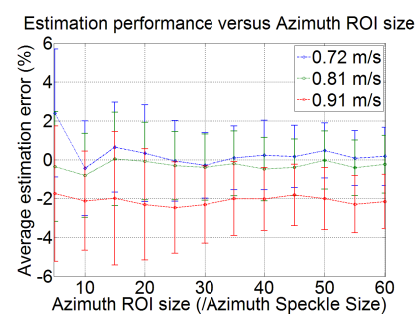

(b)

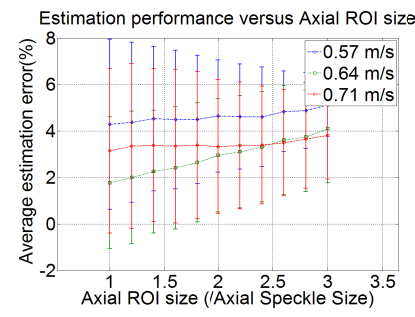

(d)

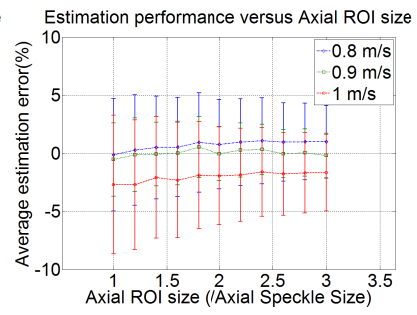

(f)
Fig.3. The relationship between estimation error and (a) lateral ROI size at $r=1.35 \mathrm{~mm}$, (b) lateral ROI size at $r=0.8 \mathrm{~mm}$, (c) lateral ROI size at $r=$ $0 \mathrm{~mm}$, (d) axial ROI size at $r=1.35 \mathrm{~mm}$, (e) axial ROI size at $\mathrm{r}=0.7 \mathrm{~mm}$, (f) axial ROI size at $r=0 \mathrm{~mm}$.

A similar analysis was done on the flow data which included random scatterer movement. These results can be seen in Figs. 4, 5 and 6. Fig. 4 shows the relationship between the estimation error and ROI size in the plug flow with random scatterer movement. Fig. 5 shows the relationship between the



(a)

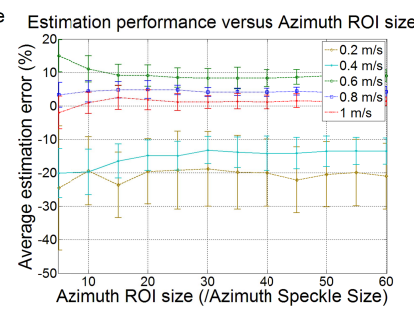

(b)
Fig.4. The relationship between estimation error and (a) axial ROI size, (b) lateral ROI size. Plug flow with random scatterer movement.

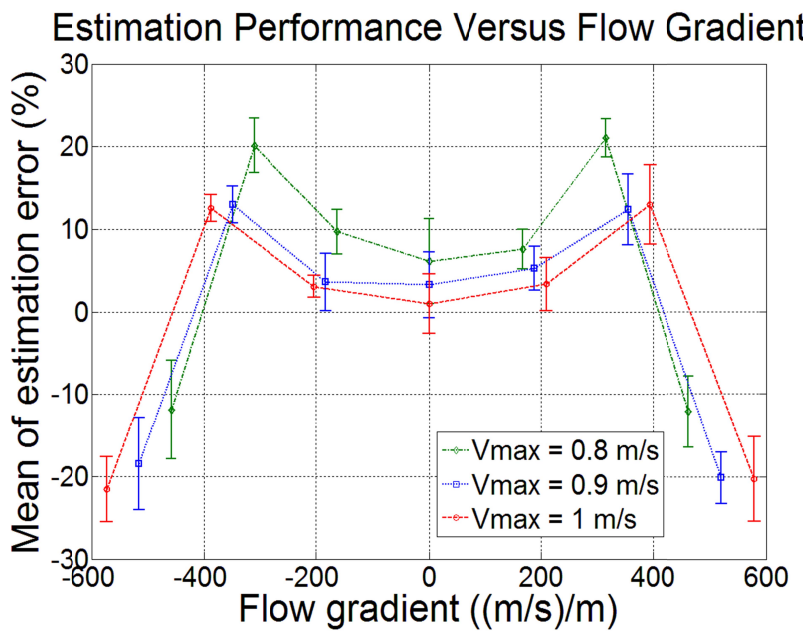

Fig.5. The relationship between estimation error flow gradient. Parabolic flow with random scatterer movement.

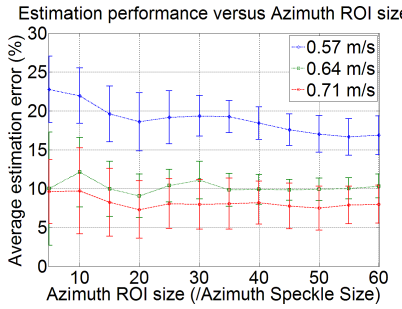

(a)

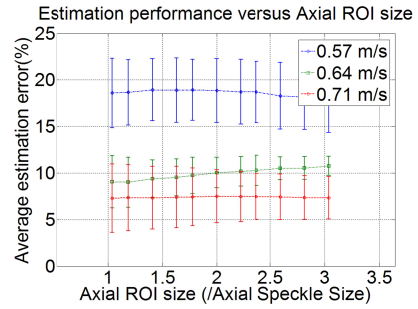

(b)
Fig.6. The relationship between estimation error and (a) lateral ROI size at $r=1.35 \mathrm{~mm}$, (b) axial ROI size at $r=1.35 \mathrm{~mm}$, Parabolic flow with random scatterer movement.

estimation error and flow gradient in the parabolic flow with random scatterer movement and Fig. 6 shows the relationship between the estimation error and ROI size in the parabolic flow with random scatterer movement.

\section{DISCUSSION}

The results in Fig. 1 show that the estimation error and its standard deviation decrease with increasing ROI size. It can be seen that the average estimation error is significant when the ROI size is small. Then the estimation error decreases when the ROI size is increasing. The reason for this is that a larger ROI includes more speckle pattern, in which the correlation properties can be better preserved for speckle size estimation. Furthermore, there is an optimal value for the ROI size both axially and laterally. In the axial direction, the estimation performance approaches an asymptote when the axial ROI size 
is about 2.5 axial speckle cell widths. In the lateral direction, the estimation performance approaches an asymptote when the lateral ROI size is about 30 lateral speckle cell lengths. The reason for this is that sufficient correlation information has been included in the ROI when the ROI size reaches these optimal values, thus, no more improvement of the estimation error can be attained afterwards.

Fig. 2 shows the relationship between the estimation error and flow gradient. It can be seen that for three different parabolic flow profiles, the average estimation error increases with increasing flow gradient, the minimum error occurs at the center of the vessel where no flow gradient is present and the estimation error increases towards the boundary of the vessel. One reason for this is that the flow gradient deceases from the boundary to the center of the vessel. Fig. 3 shows the relationship between the estimation error and ROI size with a flow gradient. In part (a), (b) and (c), it can be seen that the estimation error decreases with increasing lateral ROI size, which is similar as the result in Fig. 1(b), since more correlation information is included in a larger ROI. Also, an optimal threshold value exists for the lateral ROI size, which is around 35 lateral speckle cell lengths. However, in part (d), (e) and (f), which represent the relationship between estimation error and axial ROI size, the estimation error increases with the axial ROI size, instead of approaching an asymptote after an optimal value. We reason that increasing axial ROI size will introduce more flow gradient into the ROI and degrade the correlation information for speckle size estimation.

Fig. 4 shows the relationship between estimation error and ROI size when the random scatterer movement is presented in the plug flow. It can be seen that the average estimation error is higher than the result shown in Fig. 1 where no random scatterer movement is presented in the plug flow. The reason for this is that the random scatterer movement decreases the speckle size, thus introducing error into speckle size estimation.

Fig. 5 and 6 is similar as Fig. 2 and 3. However, the average estimation error for each blood flow profile is around three times larger when the random scatterer movement is included. These results may help interested users select optimum conditions for blood flow measurement using speckle size estimation.

\section{CONCLUSION}

This paper investigated the relationship between blood flow velocity estimation performance based on speckle size estimation and flow gradient, ROI size and random scatterer movement. Our results showed that an optimal ROI size exists both in the lateral and axial directions in plug flow, which is about 2.5 axial speckle cell widths axially and 30 lateral speckle cell lengths laterally. An optimal lateral ROI size still exists given the presence of a flow gradient; however, in the axial direction, the estimation error increases with axial ROI size because of introduced decorrelation. Similar results are shown when random scatterer movement is introduced into the blood flow; however, the average estimation error is about three times that from blood flow without random scatterer movement.

\section{REFERENCES}

[1] Hein, I. A., O'Brien and W.D., Jr., "Current time-domain methods for assessing tissue motion by analysis form relected ultrasound echo-a review," IEEE Trans. Ultrason., Ferroelect., Freq. Contr., Vol. 40, no. 2, pp. 84-102, 1993.

[2] Tiantian Xu and Gregory R. Bashford, 'Resolving the lateral component of blood flow velocity based on ultrasound speckle size change with scan direction and speed,' $31^{\text {st }}$ Annual International Conference of the IEEE Engineering in Medicine and Biology Society, Minneapolis, Minnesota, USA, pp. 491-194.

[3] Tiantian Xu and Gregory R. Bashford, "Lateral blood flow velocity estimation based on ultrasound speckle size change with scan velocity," IEEE Trans. Ultrason., Ferroelect., Freq. Contr., Vol. 57, no. 12, pp. 2695-2703, 2010.

[4] C. Kasai, k. Namekawa, A. Koyano, and R. Omoto, "Real-time twodimensional blood flow imaging using an autocorrelation technique," IEEE Trans. Sonics Ultrason., vol. 32, pp. 458-463, 1985.

[5] J. A. Jensen, Estimation of Blood Velocities using Ultrasound: A Signal Precessing Approach. Cambreidge, UK: Cambridge University Press, 1996.

[6] V. L. Newhouse, J. Reid, "Invariance of Doppler bandwidth with flow axis displacement," IEEE Ultrasonics Symposium, Honolulu, 1990, pp. 1533-1536.

[7] Martin E. Anderson, "Multi-Dimension velocity estimation with ultrasound using spatial quadrature," IEEE Trans. Ultrason. Ferroelectr. Freq. Control, vol. 45, no. 3, pp. 852-861, 1998.

[8] J. A. Jensen and P. Munk, "A new method for estimation of velocity vectors, "IEEE Trans. Ultrason. Ferroelectr. Freq. Control, Vol. 45, no. 3, pp. 837-851, 1998.

[9] Gregg E. Trahey, John W. Allison, Olaf T. Von Ramm, "Angle independent ultrasonic detection of blood flow, " IEEE Trans. Biomedical Enginnering, vol, BME-34, no. 12, pp. 964-967, 1987.

[10] Gregory Bashford, Olaf T. Von Ramm, "Ultrasound three-dimensional velocity measurements by feature tracking, "IEEE Trans. Ultrason. Ferroelectr. Freq. Control, vol. 43, no. 3, pp. 376-384, 1996

[11] Johnny Kuo, Olaf T. Von Ramm, "Three-dimensional moton measurement using feature tracking, " IEEE Trans. Ultrason. Ferroelectr. Freq. Control, vol. 55, no. 4, pp 800-810, 2008

[12] Reza Zahiri-Azar, Septimiu E. Salcuden, "Time-delay estimation in ultrasound echo signals using individual sample tracking," IEEE Trans. Ultrason. Ferroelectr. Freq. Control, vol. 55, no. 12, pp 2640-2650, 2008

[13] Malene Schlaikjer, Jorgen Arendt Jensen, "Maximum likelihood blood velocity estimator incoporating properties of flow physics," IEEE Trans. Ultrason. Ferroelectr. Freq. Control, vol. 51, no. 1, pp 80-92, 2004

[14] J. A. Jensen, "Medical ultrasound imaging," prog. Biophys, Mol. Biol., vol. 93, no. 1-3, pp. 153-165, 2007.

[15] M. F. Insana, "ultrasonic imaging," in Wiley Encyclopedia of Biomedical Engineering, m. Akay, Ed., Hoboden, NJ: Wiley, 2006.

[16] D. J. Hamilton, L. Y. L. Mo, and G. R. Bashford, "Ultrasound based quantitative motion measurement using speckle size estimation," U.S. Patent 6318179 B1, Nov. 20, 2011.

[17] Tiantian Xu and Gregory R. Bashford, "Further progress on lateral flow estimation using speckle size variation," in Proc. 2009 IEEE Trans. Sonics Ultrason., vol, 30, no. 3, pp. 156-163, 1983.

[18] J. A. Jensen and N. B. Svendsen, "Calculation of pressure fields from arbitarily shaped, apodized, and excited ultrasound transducers," IEEE Trans. Ultrason. Ferroelectr. Freq. Control, vol. 39, pp. 262-267, 1992.

[19] J. A. Jensen, "Field: A program for simulating ultrasound systems," Med. Biol. Eng. Comput., vol. 34, suppl. 1. pp. 351-353, 1996.

[20] Christoph B. Burchhardt, 'Speckle in ultrasound B-mode scans,' IEEE Trans. Sonics. Ultrason, vol. SU-25, no. 1, pp. 1-6, 1978.

[21] A. Azhim, M. Katai, et al, "Blood flow velocities in common carotid artery changes with age and exercise study by using of telemetry method," Inernational conferrence on Biomedical and Pharmaceutical Engineering, 2006, pp. 523-530. 